\section{OC-011 IS COFFEE GROUND VOMITING IMPORTANT? FINDINGS FROM A LARGE BLEEDING UNIT DATABASE WITH OUTCOMES AT 30 DAYS}

JR Schneider*, JM Thomson, A Fraser, B Vijayan, JS Leeds. Gastroenterology, Aberdeen Royal Infirmary, Aberdeen, UK

\subsection{6/gutjnl-2014-307263.11}

Introduction Upper gastrointestinal bleeding (UGIB) is a common reason for admission and "coffee ground vomiting" (CGV) has classically been considered a sign but clinical experience hasn't always borne this out. There is a paucity of data concerning endoscopic findings and outcomes in patients presenting with CGV. The aim of this study was to analyse endoscopic yield and outcomes at 30 days in patients presenting with CGV alone compared to those with haematemesis or melaena.

Methods The Aberdeen bleeding unit opened in 1991 and has recorded demographics, presenting symptoms, endoscopic diagnosis and outcomes on all admissions. Endoscopic diagnostic groups were varices, gastric ulcers (GU) or duodenal ulcers (DU), cancer, trivial (e.g. Mallory-Weiss tear (MWT)), no source found, no bleed and colonic. Analysis was performed over the period 1991 to 2005 and three groups identified; CGV alone (group A), haematemesis (group B) and melaena (group C). Endoscopic diagnosis, rebleeding and mortality rate were calculated and using group A as reference, odds ratios calculated (shown in brackets).

Results 6105 patients were admitted over the study period with suspected UGIB ( $\mathrm{A}, \mathrm{n}=1708$ (923 males), $\mathrm{B}, \mathrm{n}=1663$ (968 males) and $C, n=2734$ (1640 males)). Trivial, no source found and no bleed diagnoses were found in 1390 (75\%) group A, 1291 $(50 \%)$ group B and 1130 (40\%) group C. Group B was younger than groups $\mathrm{A}$ and $\mathrm{C}$ (mean age 50.4 vs. 64.6 and 64.8 respectively, $\mathrm{p}<0.001)$ therefore groups were stratified into $<50$ or $\geq$ 50 . In $<50$, group B had significantly more varices (OR 2.3) and MWT (OR 2.8) whereas group C had significantly more GU (OR 2.6), DU (OR 9.9) and colonic bleeds (OR 6.9). Only 4 cases had upper GI cancer all presented with melaena. 30 day mortality $(<50)$ for groups A, B and C was $2.7 \%, 1.6 \%$ and $1.9 \%$ but rebleeding was significantly higher in group C (OR 3.5$)$. In $\geq 50$, group B had significantly more varices (OR 6.9), GU (OR 1.8), gastric cancer (OR 2.4), oesophageal cancer (OR 3.2) and MWT (OR 3.1) whereas group $C$ had significantly more varices (OR 2.9), GU (OR 2.5), DU (OR 3.7), gastric cancer (OR 2.0), and colonic bleeds (OR 6.1). 30 day mortality ( $\geq 50$ ) for groups A, B and $\mathrm{C}$ was $11.8 \%, 12.5 \%$ and $11.1 \%$ but rebleeding was significantly higher in groups B and C (OR 5.3 and OR 5.0 respectively). Conclusion Presenting with CGV is associated with the same mortality as haematemesis or malaena but has significantly lower endoscopic yield and rebleeding suggesting a non-gastrointestinal cause. CGV should not be synonymous with UGIB and needs to be considered for investigations other than endoscopy.

Disclosure of Interest None Declared.

\section{OC-012 ENDOBARRIER: A BRIDGE TO SURGERY IN MORBIDLY OBESE PATIENTS?}

${ }^{1} S$ Chakravartty*, ${ }^{1} \mathrm{D}$ Sarma, ${ }^{2} \mathrm{~A}$ Miras, ${ }^{3} \mathrm{~B}$ Hayee, ${ }^{1} \mathrm{~A}$ Patel. ${ }^{1}$ Surgery, King's College Hospital, London, UK; ${ }^{2}$ Metabolic Medicine, Imperial College London, London, UK; ${ }^{3}$ Gastroenterology, King's College Hospital, London, UK

\subsection{6/gutjnl-2014-307263.12}

Introduction Bariatric surgery is the best available intervention for weight reduction in morbidly obese patients. Although outcomes are not quite comparable to surgery, an endoscopically implanted device called the Endobarrier (Endoscopic duodenojejunal bypass liner) can lead to significant weight loss and improvement of glycaemic control when compared to either dietary caloric restriction or sham procedures. Surgery may be complicated in high-risk groups like patients with poorly controlled diabetes, multiple co-morbidities or a body mass index (BMI) $>50 \mathrm{~kg} / \mathrm{m}^{2}$. We aimed to analyse the effect of an Endobarrier as an effective risk reducing tool in such high-risk patients prior to surgery.

Methods All patients who underwent an Endobarrier insertion between January and October, 2013 were analysed. This included patients with poorly controlled diabetes, patients with a BMI $>50 \mathrm{~kg} / \mathrm{m}^{2}$ or those who were at a high risk of peri-operative complications based on obesity surgery mortality risk score (OSMRS: normal score 1-5). Patient outcomes which included length of stay, complications, weight loss, and impact on glycaemic control were prospectively observed.

Results The Endobarrier was inserted in 18 patients $(13 \mathrm{M})$ with median age of 53 years (range 32-68), median weight 159 $\mathrm{kg}$ (range 102-237), median BMI $54 \mathrm{~kg} / \mathrm{m}^{2}$ (range 37-70.4) and a median OSMRS of 4 (mortality risk $=2.4 \%$ ). Device insertion was successful in all patients with no early removals required to date. The median length of stay was 1 day (range 1-5). There were no major complications apart from a single case of upper GI bleed 3 months later, which settled conservatively. Median weight over 3-6 months was now reduced to $147 \mathrm{~kg}$ (range $77-$ 192) with median weight loss of $16.2 \mathrm{~kg}(6.9-41 \mathrm{~kg})$ and 20.4 $\mathrm{kg}(9-47 \mathrm{~kg})$ at 3 and 6 months respectively. Post-procedure, diabetic medication were either decreased $(n=9 / 11)$ or no longer required $(n=2 / 11)$ in diabetic patients and at 3 months HbA1C levels improved significantly from a median of 8.7 to $6.7 \%(\mathrm{p}=0.01)$.

Conclusion Endobarrier may be used as a stepping stone to surgery in high risk patients by safely and effectively reducing weight and improving glycaemic control.

Disclosure of Interest None Declared.

\section{OC-013 KNIFE ASSISTED RESECTION (KAR) OF LARGE AND REFRACTORY COLONIC POLYPS AT A WESTERN CENTRE: FEASIBILITY, SAFETY AND EFFICACY STUDY TO GUIDE FUTURE PRACTICE}

R Bhattacharyya*, G Longcroft-Wheaton, $P$ Bhandari on behalf of Portsmouth research group. Gastroenterology, Portsmouth Hospitals NHS Trust, Cosham, Portsmouth, UK

\subsection{6/gutjnl-2014-307263.13}

Introduction ESD enables large lesions to be resected en bloc. This reduces recurrence, but ESD is technically challenging with high complication rates and hence not widely practiced in the west.

We have used a novel Knife Assisted Resection (KAR) technique.

We aim to evaluate the outcome of KAR in the treatment of large and refractory colonic polyps and identify polyp features that can predict complications and recurrence after KAR.

Methods Cohort study of patients referred to our centre for resection of refractory polyps. All patients who had KAR of colonic polyps $>20 \mathrm{~mm}$ in size from 2006 to Feb 2013 were included. All procedures were performed by a single experienced endoscopist.

The technique starts with submucosal (SM) injection followed by mucosal incision using a dual knife (Olympus KD-650L). 